\title{
An Artificial Immune Algorithms Apply to Pre-processing Signals
}

\author{
Mariusz Święcicki ${ }^{1}$, Wiesław Wajs ${ }^{2}$, and Piotr Wais ${ }^{3}$ \\ ${ }^{1}$ Institute of Computer Modelling \\ Cracow University of Technology, Kraków, Poland \\ mswiecic@pk.edu.pl \\ ${ }^{2}$ Institute of Automatics \\ University of Mining and Metallurg, Kraków, Poland \\ wwa@ia.agh.edu.pl \\ ${ }^{3}$ The State Higher School of Vocational Education in Krosno \\ waisp@poczta.onet.pl
}

\begin{abstract}
Over the past few decades there has been a growing interest in the use of biology as a source of inspiration for solving computational problems. The motivation of this field is primarily to extract useful metaphors from natural biological systems, in order to create effective computational solutions to complex problems in a wide range of domain areas. The more notable developments have been the neural networks inspired by the working of the brain, and the evolutionary algorithms inspired by neo-Darwinian theory of evolution. This paper presents the theory of an immune network model, and it tries to apply to solve signal classification problems.
\end{abstract}

\section{Introduction to Architecture Human Immune System}

The immune system of vertebrates is composed of a great variety of molecules, cells, and organs spread all over the body. There is no central organ controlling the functioning of the immune system. The main task of the immune system is to survey the organism in the search for malfunctioning cells from their own body (e.g., cancer and tumour cells), and foreign disease causing elements (e.g., viruses and bacteria). Every element that can be recognised by the immune system is called an antigen (Ag). The cells that originally belong to our body and are harmless to its functioning are termed self (or self antigens), while the disease causing elements are named nonself (or nonself antigens). The immune system, thus, has to be capable of distinguishing between what is self from what is nonself; a process called self/nonsel discrimination, and performed basically through pattern recognition events. The main functions of the $\mathrm{B}$ cells include the production and secretion of antibodies $(\mathrm{Ab})$ as a response to exogenous proteins like bacteria, viruses. Each B cell is programmed to produce a specific antibody. The antibodies are specific proteins that recognize and bind to another particular protein. The production and binding of antibodies is usually a way of sending signal for other cells to kill or remove the bound substance. 


\section{An Artificial Immune Network for Signal Analysis}

Main goal our system is classification signals so there are some problems that have to solve. The first problem is connected with algorithm of learning immune network. The next problem is related to structures of data, which are responsible for representation of signals. Solutions of these problems are presented below. The last paragraph shows result of classification signals by our immune network.

The signal comes inputs system, is interpreted as antibody ( $\mathrm{Ab}$ ) so task of immune network is found antigen Ag that will be suitable for Ab. The Ag-Ab representation will partially determine which distance measure shall be used to calculate their degree of interaction. Mathematically, the generalized shape of a molecule $(m)$, either an antibody $(\mathrm{Ab})$ or an antigen $(\mathrm{Ag})$, can be represented by a set of real-valued coordinates $m=\langle m 1, m 2, \ldots, m L>$, which can be regarded as a point in an $L$-dimensional real-valued space.

$$
D=\sqrt{\sum_{i=1}^{L}\left(a b_{i}-a g i\right)^{2}}
$$

The affinity between an antigen and an antibody is related to their distance that can be estimated via any distance measure between two vectors, for example the Euclidean or the Manhattan distance. If the coordinates of an antibody are given by $<a b 1, a b 2$, $\ldots, a b L>$ and the coordinates of an antigen are given by $<a g 1, a g 2, \ldots, a g L>$, then the distance $(D)$ between them is presented in equation (1), and that use real-valued coordinates and that measure distance are called Euclidean shape-spaces.

In this article, we based on algorithm that was proposed in paper [1, 3] by de Castro and Von Zuben. This learning of immune net algorithm was adapted to classifying signals. Below this algorithm is presented. The learning algorithm lets building of set that recognizes and represents the data structural organization. The more specific the antibodies, the less parsimonious the network (low compression rate), whilst the more generalist the antibodies, the more parsimonious the network with relation to the number of antibodies. The suppression threshold controls the specificity level of the antibodies, the clustering accuracy and network plasticity.

For each Agj $\in$ Ag do

Determine its affinity fi,j, $i=1, \ldots, N$, to all Abi. fi, $j=1 / D i, j, i=1, \ldots, N$

A subset $A b\{n\}$ contains the $n$ highest affinity antibodies is selected;

The $n$ selected antibodies are going to clone proportionally to their antigenic affinity

The set $C$ is submitted to a directed affinity maturation process generating a mutated set $C^{*}$

Determine the affinity $d k, j=1 / D k, j$ among Agj and all the elements of $C^{*}$ :

From $C^{*}$, re-select $\zeta \%$ of the antibodies with highest put them into matrix of clonal memory;

Apoptosis: eliminate all the memory clones from $M j$ whose affinity $D k, j>$ od:

Determine the affinity si, $k$ among the memory clones: 
Clonal suppression: eliminate those memory clones whose si, $k<\sigma$ :

Concatenate the total antibody memory matrix with the resultant clonal memory $M j$

Determine the affinity among all the memory antibodies from $A b\{m\}$ :

Network suppression: eliminate all the antibodies such that si, $k<\sigma$ :

\section{Result of Experiments}

This part of our paper includes results of which will be discussed. As was mentioned, we tried to construct system, which was able to classifying signals. Our experiments will consist of two phases, the first phase is phase of learning process of immune network. The second phase is phase of testing process. During Phase of testing, the signals will contain noise so it will make that process of recognizing will be either difficult or even impossible. For that reason the trained artificial immune net have to be cable of generalizing of signals. All Samples of signals have the same number of values. A Distance between either $\mathrm{Ag}$ and $\mathrm{Ab}$ or $\mathrm{Ab}$ and $\mathrm{Ab}$ will be counted by using the equation (1). A Set, which is used for training artificial immune net, includes patterns of signals. As was mentioned, in our case, the samples of signals that are included will be interpreted as antigens during the teaching process by having been trained net. A first task of system will be classification of samples, which belong to family of functions that are described by equations (2).

$$
\begin{aligned}
& f_{1}(t, i)=\sin (i \cdot x) \quad f_{2}(t, i)=\operatorname{sign}(\sin (i \cdot x)) \\
& \text { where }: i \in[0.2,1.2] ; \quad t \in[0,5 \cdot \pi]
\end{aligned}
$$

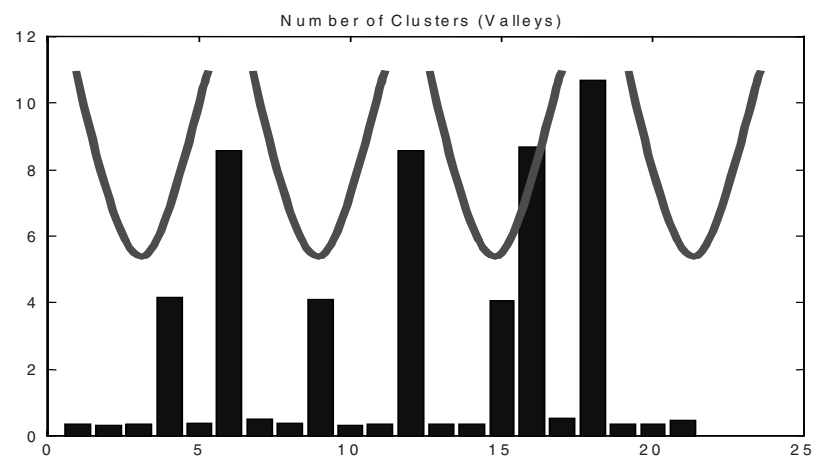

Fig. 1. Number of clusters (Peaks +1 , or Valleys) for this MST

There are 60 samples subdivided into 4 clusters (non-overlapping classes). Fig. 1 depict result of training of the immune network. Each of classes contains 6 cells. The net had following training parameters: $n=4, \zeta=0.2, \sigma d=1.0, \sigma s=0.001$ and $d=10$. 
The stopping criterion is a fixed number of generations: Ngen $=200$. The resulting network contains 22 cells. The suppression threshold ( $\sigma s)$ controls the specificity level of the antibodies, the clustering accuracy and network plasticity. In order to provide the user with important information on how to set up the immune network algorithm parameters.

The change of parameters algorithm have influence on sensitivity analysis of the algorithm. An Especially, the $\sigma s$ suppression threshold is responsible for sensitivity work of the immune net.

\subsection{A Phase of Testing of Artificial Immune Net}

On input of the trained immune net will be delivered samples of signals don't belong to the learning set. The samples of signals were intentionally deformed by added noise. In the Fig. 2 is result of the immune net answer. The dashed line presents values of input sample. The continuous line shows the best answer of our net. The algorithm that was described in the third paragraph, returns a queue of possible answer. The queue is ordered and first place in the queue is occupied by vector of signal values that are connected with class that is matched an input signal.

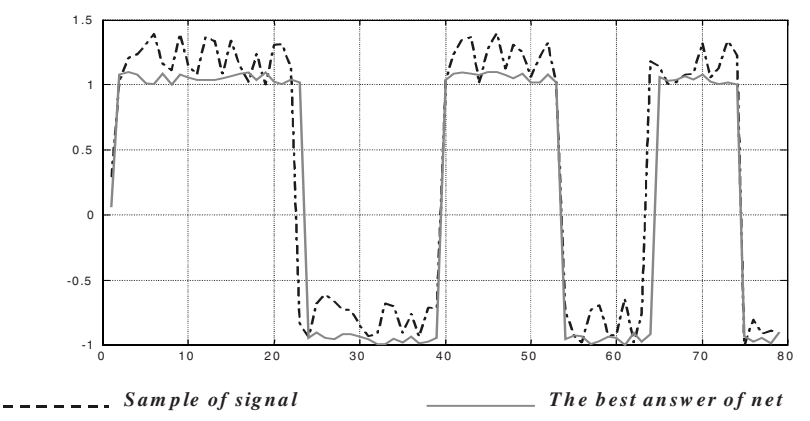

Fig. 2. Result of process classification input signal

As we can see, the Fig.2 shows very interesting case, because although the input signal was very deformed, the artificial immune network correctly classified it. That is mean that the net is able to generalization of input signals.

\section{Conclusion}

This paper described mechanics of immune network in human organism. In this paper is included describing positive selection. It is known that these the phenomenon are used for building of artificial immune systems.. The model is connectionist in nature but it follows an evolutionary-like learning algorithm that is the immune clonal selec- 
tion principle. Finally, there was presented model of application that based on immune network theory. The application was solving problems of identification signals.

\section{References}

1. De Castro, L. N., Von Zuben, F. J. (2000a), An Evolutionary Immune Network for Data Clustering, Proc. of the IEEE SBRN, pp. 84-89.

2. De Castro, L. N., Von Zuben, F. J. (2000b), The Clonal Selection Algorithm with Engineering Applications, GECCO’00 - Workshop Proceedings, pp. 36-37.

3. De Castro, L. N., Von Zuben, F. J. (1999), Artificial Immune Systems: Part I - Basic Theory and Applications, Technical Report - RT DCA 01/99, p. 95. 